\title{
A Group-Based Yule Model for Bipartite Author-Paper Networks
}

\author{
Michel L. Goldstein * Steven A. Morris $\$$ and Gary G. Yent \\ Oklahoma State University \\ Electrical and Computer Engineering \\ Stillwater, OK 74078, USA
}

(Dated: October 24, 2018)

\begin{abstract}
This paper presents a novel model for author-paper networks, which is based on the assumption that authors are organized into groups and that, for each research topic, the number of papers published by a group is based on a success-breeds-success model. Collaboration between groups is modeled as random invitations from a group to an outside member. To analyze the model, a number of different metrics that can be obtained in author-paper networks were extracted. A simulation example shows that this model can effectively mimic the behavior of a real-world author-paper network, extracted from a collection of 900 journal papers in the field of complex networks.
\end{abstract}

* mgoldst@okstate.edu

isamorri@okstate.edu

tgyen@okstate.edu 


\section{INTRODUCTION}

This paper presents a realistic bipartite model of author-paper networks, a phenomenon which has been studied since the 1920s 1]. The proposed growth model is based on modeling groups of authors using a 'nested' Yule process [2], and further models 'loose ties' among author groups as a Watts-Strogatz small world process [3]. The full bipartite representation of the network allows construction of many meaningful metrics to evaluate the validity of the proposed model against actual author-paper networks. Using a collection of 900 papers covering the topic of complex networks, we will show that the proposed model faithfully reproduces the characteristics of six metrics: 1) authors per paper distribution, 2) papers per author distribution (Lotka's Law), 3) co-author clustering coefficient distribution, 4) co-authorship per author pair distribution, 5) collaborator per author distribution, and 6) minimum path between author pairs distribution.

The model and the validation metrics presented in this paper are innovative when considered against previous models of Lotka's Law or models of author collaboration networks. Lotka's Law models deal with single authors without modeling collaboration, while collaboration models cannot describe Lotka's Law and single authors. Both types of models are usually validated against simple power law link degree distributions: papers per author for testing Lotka's Law models, or collaborators per author for testing author collaboration models. Power law link degree distributions are easy to duplicate using several types of pro-

cesses [4]. Because of this, such simple models offer little insight into underlying processes that generate author-paper networks.

The proposed model, which deals with groups of authors rather than single authors, reveals the importance of research workgroups (author groups) in author-paper networks. The model indicates that publication by author groups is driven by a success-breeds-success (SBS) process, and further, that authorship by single authors within these groups is a SBS process as well. Yet, surprisingly, intergroup collaboration, i.e. loose ties, appears to be well modeled by a small world network of random interlinkages.

Bipartite author-paper networks are formed by two types of entities, the authors and papers, and the authorship links between them. There exists much analysis in the literature on the features of real-world author-paper networks. The first of these analyses were presented by Lotka 1]. His analysis, which contained a dataset of journal articles compiled 
by hand, showed that the distribution of the number of papers per author follows a zeta distribution, a pure power-law, with an exponent of approximately 2. This observation is currently referred to as the Lotka's Law of Scientific Productivity. A large number of other studies reinforced the power-law concept for the number of papers per author distribution, especially when considering only the tail of the distribution. These studies show that the observed exponent varies with the dataset [5], $[6]$.

The observation of this distribution is very important, but it does not explicitly provide an insight into network dynamics. For this, a dynamic growth model is needed. Of the dynamic models in the literature, almost all are evaluated using crude comparisons to simple paper per author distributions and ignore other important metrics, such as clustering coefficient distribution or collaborator distribution. A complete and useful model must be able to mimic the real behavior of the author-paper network across many important network metrics.

This paper provides a new model for the growth of author-paper networks and a stepby-step presentation of the important features of a real-world author-paper network that a model has to mimic. The proposed model, although very simple, approximates well all these features, thus building confidence in the validity of the model and the insight that the model provides into the actual dynamics of real-world author-paper networks.

\section{AUTHOR-PAPER NETWORK MODELS}

A number of different bipartite author-paper models exist in the literature. These models attempt to explain the process generating the power-law distribution of the papers per author distribution. They are fundamentally different from the usual preferential connection models, such as the Barabási-Albert model [4], because they model bipartite networks, in which one partition contains all authors and the other all papers. Although it is possible to transform a bipartite network into a simple graph by projection [7], this transformation removes the ability to calculate metrics to evaluate the validity of the model.

In the model presented by Newman et al. [8], the goal was to enable the generation of any degree distributions, such as Poisson, exponential and power-law, for simple, directed and bipartite graphs. The proposed method is very general but it is mainly focused on predicting three features: the average degree, the clustering coefficient [3] , and the degree distribution of the projected graph. The model is able to effectively predict the features for 
a network of company directors, however it fails to approximate the features of authorship networks.

Huber [9] presents a model of authors to predict five different features: the rate of production, career duration, randomness, Poisson-ness distribution (related to the variance of the author's productivity through time) and the distribution of papers per author. Huber's model is complex and involves distributions of career durations (assumed exponential) and Poisson distributed counts of papers, based on the author's productivity. Although this model predicts very well the features of interest, its major drawback is that it does not model the existence of co-authors. In the model, each author is "evolved" individually. A useful model must have the ability to predict collaboration patterns.

Recently, Börner et al. [10] presented a model in which the author network and the reference networks evolve simultaneously. This study is an important acknowledgement that multiple interconnected networks exist in collections of journal papers, and that the challenge of modeling such paper collections is to find the basic rules of author behavior that produce the growth characteristics of the multiple interconnected networks contained in them. Börner et al's main goal was to predict the evolution of the number of papers, authors and citations in a large and heterogeneous collection of journal articles, such as all of the papers published in the Proceedings of the National Academy of Science from 1981 to 2001. The paper includes a detailed set of proposed author behavioral rules and predicts gross measures of author, paper, and reference growth well, but the study does not discuss detailed metrics of network characterization.

One major disadvantage of all models found in the literature is the inability to predict most of the features of real-world networks. The prediction of only one or two features greatly weakens the usefulness of such models as models of real-world behavior.

\section{PROPOSED GROUP-BASED YULE MODEL}

A Yule model is a preferential connection process first proposed as a model of biological evolution by Yule in 1924 [2]. Our model uses a Yule process to model the growth of author groups in the author-paper network. The proposed model is based on the observation that usually authors are part of a research group. Most of the papers they write are co-authored with other members of their group. Collaboration between research groups happens, but 


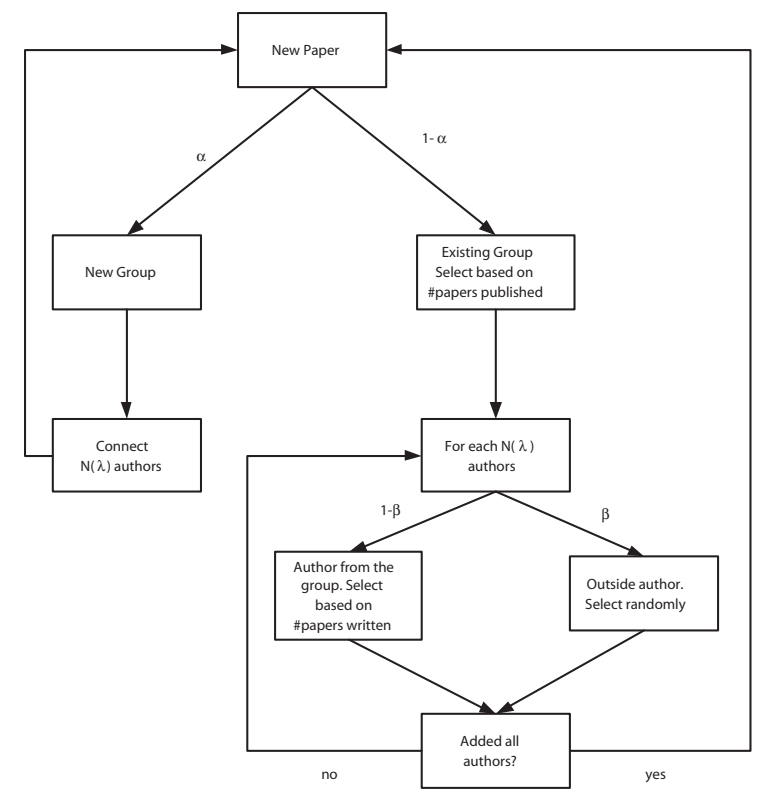

FIG. 1: Diagram of the proposed group-based Yule model for author-paper networks

multi-group papers are far less common than in-group papers.

A diagram of the model can be seen in Fig. 1. When a paper is created there is a probability $\alpha$ that a new author group is created with $N_{g}$ all new members, where $N_{g}$ is a constant. The number of authors of the paper, $N(\lambda)$, is the first author plus a Poissondistributed number of additional authors. This 1-shifted-Poisson distribution has parameter $\lambda$. The probability distribution of the 1-shifted-Poisson, $p_{s p}(k)$, is given in Eq. 11.

$$
p_{s p}(k)=\frac{\lambda^{(k-1)} e^{-\lambda}}{(k-1) !}, \quad k=\{1,2, \ldots\},
$$

where $k$ is the number of authors and $p_{s p}(k)$ is the probability of a paper having $k$ authors.

If a new group is not created, an existing author group is chosen using the following probability distribution:

$$
p_{g}(q)=\frac{q}{N_{p}}
$$

where $q$ is the number of papers that this group has published, $N_{p}$ is the total number of papers in the network, and $p_{g}(q)$ is the probability of an existing group authoring a paper. This is the Yule process which favors groups in proportion to the number of papers they have published.

When an existing group is selected, it is necessary to select the authors within the group 
that author the paper. The number of authors of the paper is modeled as a 1-shifted Poisson distribution. In order to model interconnection between groups ('loose ties'), for each author, there is a probability $\beta$ that this author is from another group. If so, the author is selected randomly from among all authors in the network, whether they have authored a paper or not. If an outside author is not chosen, an author from the selected group is chosen. This selection is done by another preferential connection process, modified to allow selection of authors that have never published a paper. The probability of selecting an author $i$ in the group is:

$$
p_{a}(i)=\frac{k_{i}+1}{\sum k_{j}+N_{g}},
$$

where $k_{i}$ is the number of papers written by author $i, \sum k_{j}$ is the sum of the number of authorships among the authors in the group and $N_{g}$ is the number of authors in the group. This is a preferential attachment process which favors authors by the number of papers they have previously published.

The paper creation cycle of Fig. 1 repeats until the desired number of papers is added to the network.

In summary, this model has four parameters: the group size, $N_{g}$, assumed always constant for this simple model; the probability of creating a new group, $\alpha$; the probability of choosing an author from another group, $\beta$; and the Poisson parameter that defines the distribution of number of authors per paper $\lambda$. Given a dataset to be modeled, it is easy to analytically determine $\alpha$ and $\lambda$.

The following section presents methods for obtaining these parameters to model a realworld network. Methods for correctly validating the model are also presented, by analyzing network metrics.

\section{EXAMPLE:}

The example is a collection of papers covering the specialty of complex networks. This data set, collected from the Science Citation Index, contains 900 papers, 1,354 authors, and 2,274 authorships linking authors to papers. The first parameter, $\alpha$, is obtained by determining the probability of new group creation. This probability is estimated using a paper-by-paper pass through the network to determine the fraction of papers that appeared 
with a completely new set of authors.

The parameter $\lambda$ is calculated by dividing the total number of authorships by the number of papers and subtracting 1 (1-shifted-Poisson). The number of authors per group, $N_{g}$, was chosen heuristically as 20 , which is assumed as the upper limit of the number of researchers that can efficiently interact as a group.

The 'loose tie' parameter $\beta$ is estimated by matching the co-authorship distribution (the distribution of the number of times pairs of authors have co-authored) by trial-and-error. The matching of the co-authorship distribution will be explained below.

The parameters estimated for the example network are:

- $\hat{\alpha}=0.33$

- $\hat{\beta}=0.1$

- $\hat{\lambda}=1.527$

- $N_{G}=20$

To validate the model, several metrics are used to compare model simulations to the actual network. The following metrics are used for comparison:

Authors per paper The distribution of the number of authors per paper. As discussed above, this is simulated as 1-shifted Poisson distributed. Note in Fig. 2 the close match of actual to simulated distributions. This metric is important because it predicts the mean number of participants on projects within the group, an important measure of interaction within workgroups.

Papers per author distribution (Lotka's Law) This is the distribution of the number of papers that each author published. Note in Fig. 3 the close match of simulated frequencies to actual frequencies for this metric. This metric is important because it measures the distribution of productivity among authors in a specialty, modeling the formation of core groups of researchers in a specialty. The inset in Fig. 3 shows the model-predicted paper per author distribution, generated by gathering statistics from 1,000 simulations. The predicted distribution certainly models Lotka's Law, producing an excellent fit to a zeta distribution with an exponent of 2.77. Fitting was done using 
Maximum Likelihood Expectation and the fit passed a Kolmogorov-Smirnov (KS) test 11] at an Observed Significance Level (OSL) of 10\% $<O S L<1 \%, T=0.0031$, $N=1.3 \times 10^{6}$.

Co-author clustering coefficient distribution The clustering coefficient was first introduced by Watts and Strogatz [3] as a scalar mean clustering coefficient. However, when observing the distribution of the clustering coefficients, a very interesting characteristic is found in co-author networks: a large spike at unity. Therefore, it is imperative to use the distribution as the metric rather than the mean, which effectively hides unity spike behavior. For example, although author networks usually have a mean clustering coefficient of 0.8 , comparable to that of citation networks [4], the distribution of the co-author networks clustering coefficient is fundamentally different from the distribution of clustering coefficient in citation networks [12]. Newman discusses this distribution in 8] and models it, with limited success. Note in Fig. 4 that simulation using the proposed model fully mimics the distribution of the clustering coefficient. This metric is important because it measures the tendency of authors to work in local groups.

Collaborator distribution The distribution of the number of unique co-authors to each author in the network. Newman attempted to model this distribution with only partial success [8]. Note the close match of the simulated to actual co-authorship frequencies in Fig. 5. This metric is important because it measures the tendency of authors to work with other authors.

Co-authorship distribution This is the distribution of the number of common papers between pairs of authors, across all author pairs in the network. Fig. 6 shows that the proposed model matches the actual distribution well. This is an important metric because it measures the tendency of pairs of authors to repeatedly work together on individual projects.

Minimum distance distribution Fig. [7 shows the distribution of the minimum distance between a pair of authors in the network, i.e. the minimum length of the path of coauthorships between them. This metric is important because it measures the tendency of groups to invite outside workers on projects. 


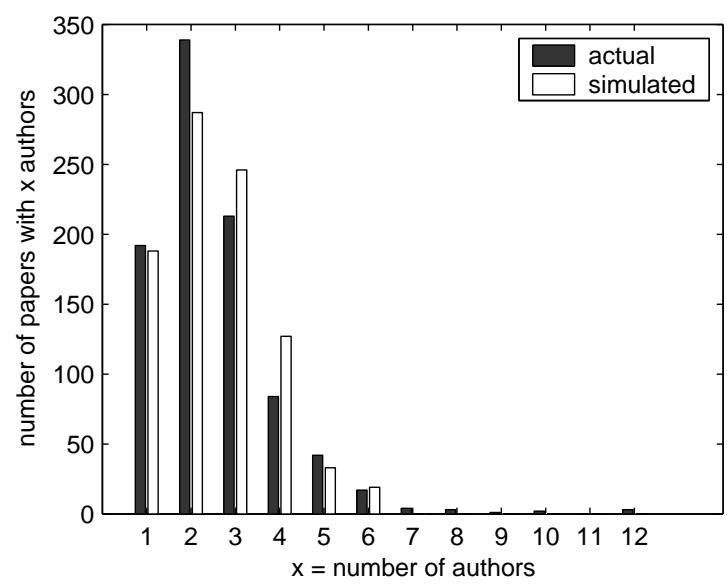

FIG. 2: Frequency distribution comparison for the number of authors per paper between the actual distribution and the simulated distribution. $\lambda_{\text {actual }}=1.527, \lambda_{\text {sim }}=1.651$.

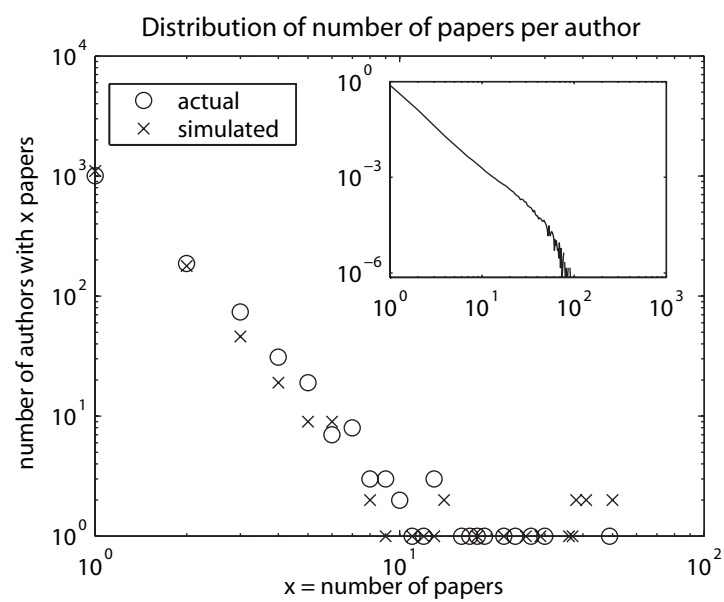

FIG. 3: Frequency distribution comparison for the number of papers per author, Lotka's Law, between the actual distribution and the simulated distribution. The actual distribution has a power-law exponent of $\gamma=2.544$ and the simulated distribution has $\gamma=2.770$. The inset shows the model-predicted paper per author distribution, which fits a zeta distribution.

For additional discussions of network metrics applicable to author-paper networks, see Newman [6], who discusses several of the metrics used here.

All metrics shown above present a close match between the real-world network and the model simulation. As an exception, the minimum path distribution shows a fair amount of deviation, but this distribution appears to be unstable and tends to change greatly from simulation to simulation. The actual minimum path length distribution is probably unstable 


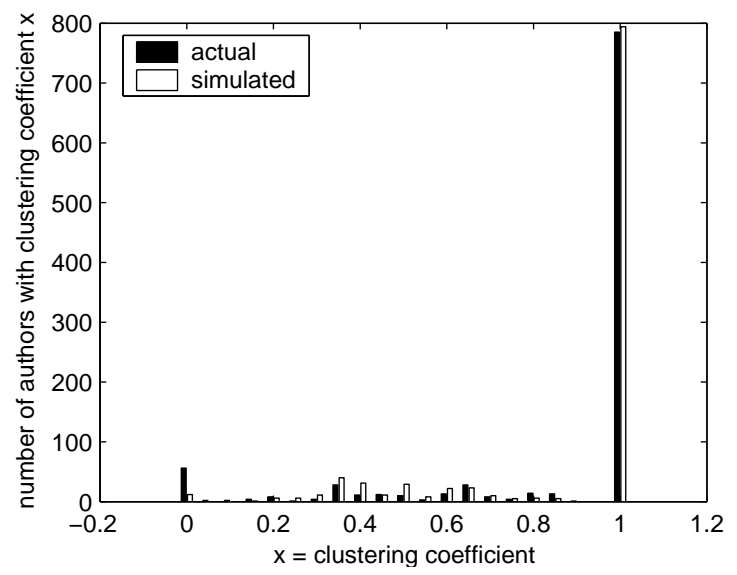

FIG. 4: Frequency distribution comparison of the clustering coefficient. $C_{\text {actual }}=0.867, C_{\text {sim }}=$ 0.881 .

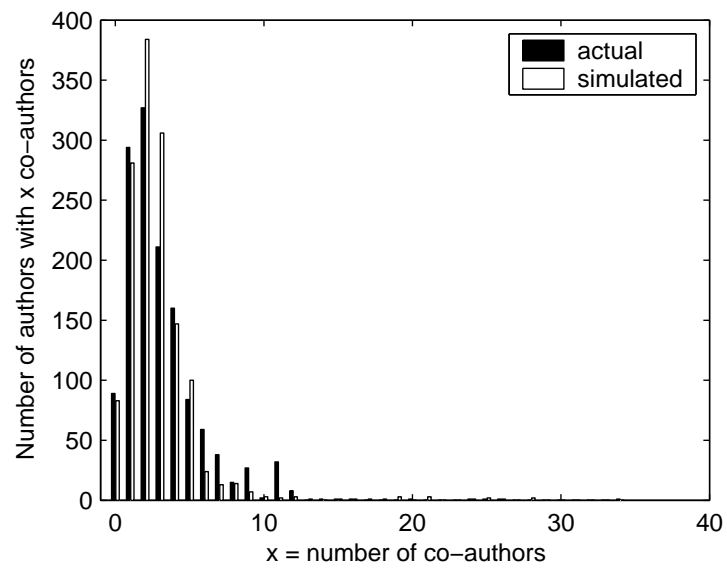

FIG. 5: Frequency distribution comparison of the number of collaborators per author. $\mu_{\text {actual }}=$ $3.15, \mu_{\text {sim }}=2.82$.

as well, but investigation of that hypothesis is outside the scope of this paper.

\section{CONCLUSIONS}

This paper proposes a very simple model for author-paper networks by introducing the concept of preferential attachment of group authoring of papers. Adding this simple concept to a Yule-type process it was possible to obtain very similar behavior using multiple metrics, when comparing to a real-world network. This suggests that, in the real world, the modeling of research groups is essential to understand the dynamics of paper authoring. Analysis of 


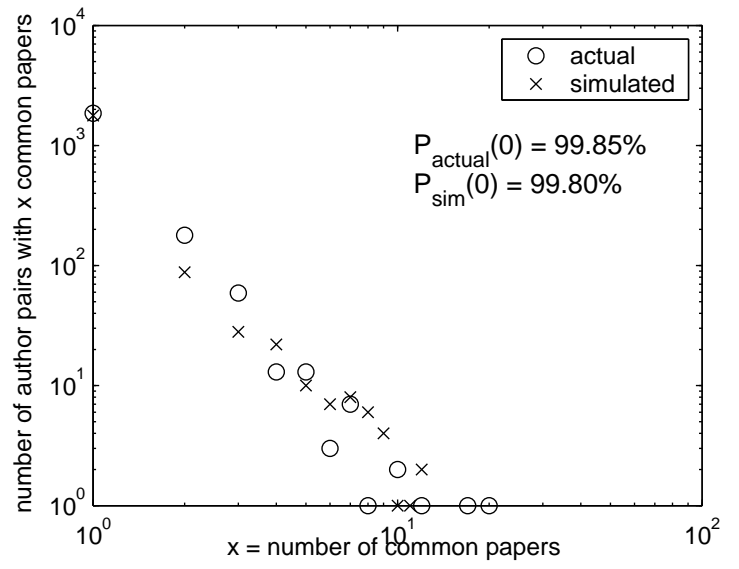

FIG. 6: Frequency distribution comparison of the co-authorship distribution, showing the number of papers co-authored by each pair of authors.

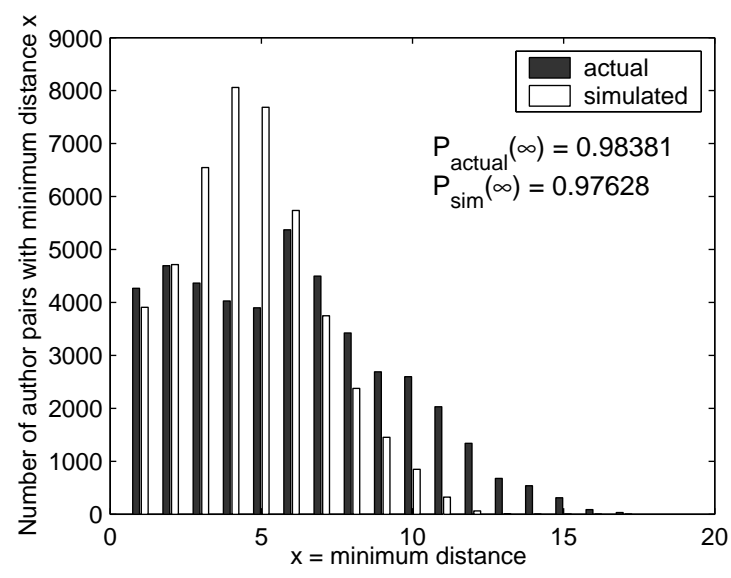

FIG. 7: Frequency distribution comparison of the minimum distance between authors, i.e. the minimum number of links between each pair of authors.

single authors or random connections between authors, as proposed by previous researchers, do not provide a reasonable model of reality.

Another important conclusion drawn from this model is that 'loose ties' between groups is well modeled by simple random inter-group co-authorships. This implies that group collaboration does not actually work by establishing formal long-term commitments, but by single collaborations, possibly from informal meetings at conferences, or e-mail discussion lists. Multiple collaboration with outside groups may happen in real life, but such collaborations are uncommon and do not affect the gross characteristics of the network. This model further implies that outside collaboration is not dependent on the amount of work that the 
outside person has done in the field.

Note that while there is local preferential connection of authors within groups, and global preferential connection of the groups themselves, the inter-group linkage approximates a Watts-Strogatz small world process. The model here is really a hybrid, being a "nested preferential connection, global small world" model.

We also showed that using only a single metric, such as the distribution of papers per author, or a single mean value for the clustering coefficient, incompletely validates a model. Analyzing multiple metrics, allows validation against specific behaviors that fully characterize the network.

It is important to note that this model only accounts for the behavior of authorships in a collection of papers. To actually understand the nature of collections of journal papers it would be necessary to implement and discuss the interaction of this author-paper bipartite network with the other bipartite networks in the paper collection, such as the paper-reference network 12, 13, 14], paper-journal network (Bradford's Law) 15], and paper-term network (Zipf's Law) [15]. The analysis of their complex interaction will certainly shed light on a large number of open questions regarding the growth and mapping of information structures.

[1] A. J. Lotka, J. Wash. Acad. Sci. 16, 317 (1926).

[2] G. U. Yule, Philosophical Transactions of the Royal Society of London, Series B 213, 21 (1924).

[3] D. J. Watts and S. H. Strogatz, Nature 393, 440 (1998).

[4] R. Albert and A.-L. Barabási, Rev. Mod. Phys 74, 47 (2002).

[5] M. L. Pao, Inf. Proc. Mngt. 21, 305 (1985).

[6] M. E. J. Newman, in Complex Networks, edited by E. Ben-Naim, H. Frauenfelder, and Z. Toroczkai (Springer, Berlin, German, 2004).

[7] S. N. Dorogovtsev and J. F. F. Mendes, Advances in Physics 51, 1079 (2002).

[8] M. E. J. Newman, S. H. Strogatz, and D. J. Watts, Phys. Rev. E 64, 026118 (2001).

[9] J. C. Huber, J. Am. Soc. Info. Sci. Tech. 53, 209 (2002).

[10] K. Borner, J. T. Maru, and R. L. Goldstone, Proc. Natl. Acad. Sci. U. S. A. 101, 5266 (2004).

[11] M. L. Goldstein, S. A. Morris, and G. G. Yen, Europ. Phys. Rev. B (in press) (2004). 
[12] S. A. Morris, J. Am. Soc. Info. Sci. Tech. (in press) (2004).

[13] S. Naranan, J. of Documentation 27, 83 (1971).

[14] S. Redner, Euro. Phys. J. B 4, 131 (1998).

[15] H. D. White and K. W. Mccain, Annual Review of Information Science and Technology 24, 119 (1989). 